\title{
Accessibility of Vendor-Created \\ Database Tutorials for People \\ with Disabilities
}

Joanne Oud

\begin{abstract}
Many video, screencast, webinar, or interactive tutorials are created and provided by vendors for use by libraries to instruct users in database searching. This study investigates whether these vendorcreated database tutorials are accessible for people with disabilities to see whether librarians can use these tutorials instead of creating them in-house. Findings on accessibility were mixed. Positive accessibility features and common accessibility problems are described, with recommendations on how to maximize accessibility.
\end{abstract}

\section{INTRODUCTION}

Online videos, screencasts, and other multimedia tutorials are commonly used for instruction in academic libraries. These online learning objects are time consuming to create in-house and require a commitment to maintain and revise when database interfaces change. Many database vendors provide screencasts or online videos on how to use their databases. Should libraries use these vendor-provided instructional tools rather than spend the time and effort to create their own? Many already do: a study shows that 17.7 percent of academic libraries link to tutorials created by third parties, mainly by vendors or other libraries. ${ }^{1}$

When deciding whether to use vendor-created tutorials, one consideration is whether the tutorials meet accessibility requirements for people with disabilities. The importance of accessibility for online tutorials has been increasingly recognized and outlined in recent library literature. ${ }^{2}$ People with disabilities make up one of the largest minority groups in the United States and Canada, and studies show that about 9 percent of university or college students have a disability. ${ }^{3}$

Problems with web accessibility have been well documented. People with disabilities are often unable to access the same online sites and resources as others, creating a digital divide. ${ }^{4}$ Even if people with disabilities can access a site, it is more difficult for many to use it. ${ }^{5}$ Assistive technologies, like screen-reading software, enable access but add an extra layer of complexity in interacting with the site, and blind or low-vision users can't always rely on visual cues to navigate and interpret sites. A recent study of library website accessibility concluded that typical library websites are not designed with people with disabilities in mind. ${ }^{6}$

Joanne Oud (joud@wlu.ca) is Instructional Technology Librarian and Instruction Coordinator, Wilfrid Laurier University, Ontario, Canada. 
Libraries, which are founded on a philosophy of equal access to information, should be concerned about online accessibility. Legal requirements for providing accessible online web content vary, but exist in every jurisdiction in the United States and Canada. Apart from the legal requirements, recent literature points out that equitable access to information for people with disabilities is a matter of human rights and an issue of diversity and social justice, and calls on libraries and librarians to improve their commitment to online accessibility. ${ }^{7}$ It is important for libraries to participate in creating level playing field and to avoid creating conditions that make people feel unequal or prevent them from equitable access.

It is unclear whether librarians can assume vendor-created instructional tutorials are accessible. Studies on vendor database accessibility have been mixed, showing some commitment to and improvements in accessibility on one hand, but sometimes substantial gaps in accessibility on the other. ${ }^{8}$ The focus until now has been exclusively on the accessibility of database interfaces. This study investigates the accessibility of online tutorials, including videos, screencasts, interactive multimedia, and archived webinars created by database and journal vendors and offered as instructional materials to librarians and patrons, to determine whether they are a viable alternative to making in-house training materials.

\section{LITERATURE REVIEW}

Although a few articles exist on how to make video tutorials accessible, ${ }^{9}$ no studies have evaluated the accessibility of already-created video or screencast tutorials. There are, however, some studies evaluating the accessibility of vendor databases. Byerley, Chambers, and Thohira surveyed vendors in 2007 and found that most felt they had integrated accessibility standards into their search interfaces, and nearly all tested for accessibility to some degree, though not always with actual users. ${ }^{10}$

These findings conflict somewhat with the results of other studies. Tatomir and Durrance evaluated the accessibility of thirty-two databases with a checklist and found that although many did contain accessibility features, 72 percent were marginally accessible or inaccessible. ${ }^{11}$ Similarly, Dermody and Majekodunmi found that students with print-related disabilities who use screen-reading software could only complete 55 percent of tasks successfully because of accessibility barriers and usability challenges. ${ }^{12}$ DeLancey surveyed vendors and examined VPATs, or product accessibility claims, and found that vendors felt they were compliant with 64 percent of US Section 508 items. ${ }^{13}$ Especially relevant to this study, only 23 percent of vendors said that the multimedia content within their products was compliant, and 46 percent admitted multimedia content was not compliant at all. Since vendor VPAT forms are completed for databases and other products only, and not the instructional tutorials created by vendors on how to use those products, vendor accessibility claims for instructional tutorials are unknown.

Although no studies have been done on the accessibility of video or screencast tutorials, some have been done on the accessibility of multimedia or other related kinds of online learning. 
Roberts, Crittenden, and Crittenden surveyed 2,366 students taking online courses at several US universities. A total of 9.3 percent of those students reported that they had a disability, and of those, 46 percent said their disability affected their ability to succeed in their online course, although most reasons cited were not related to technical accessibility barriers. ${ }^{14}$ Kumar and Owston studied students with disabilities using online learning units that contained videos. All students in the study reported at least one barrier to completing the learning units..$^{15}$ Although this study involves student use of video tutorials, it doesn't report on accessibility issues specific to those tutorials.

Previous studies of vendor products focus exclusively on database interfaces, and previous studies of online learning have not focused on screencast accessibility. Therefore this study's goal is to investigate how accessible vendor-created video tutorials are. Accessibility is defined as both technical accessibility (can people with disabilities locate, access, and use them) and usability (how easy it is for people with disabilities to use them). This study will look at which major accessibility issues there are (if any) and make recommendations on whether librarians can direct students to them rather than making in-house instructional videos.

\section{METHOD}

An evaluation checklist (see appendix 2) was developed for this study using criteria drawn from the Web Content Accessibility Guidelines (WCAG) 2.0. WCAG 2.0 is the most widely recognized web-accessibility standard internationally. Much recent accessibility legislation adopts it, including the in-process revisions to Section 508 guidelines in the United States. ${ }^{16}$ WCAG 2.0 is also consistent with tutorial accessibility best-practice advice found in recent articles, which emphasize the need for accurate captions, keyboard accessibility, descriptive narration, and alternate versions for embedded objects, among other criteria. ${ }^{17}$

The checklist has twenty items and is split into two sections, "Functionality" and "Usability." Functionality items test whether the tutorial can be used by people using screen-reading software or a keyboard only, and include whether the tutorial is findable on the page and playable, whether player controls and interactive content can be operated by keyboard, whether captions are available, and whether audio narration is descriptive enough so someone who can't see the video can understand what is happening. Usability items test how easy the tutorial is to use. Examples include clear visuals and audio, use of visual cues to focus the viewer's attention, and short and logically focused content.

To help prioritize the importance of checklist items, the local Accessible Learning Centre (ALC), which supports students on campus who use assistive technologies, was consulted about the difficulties most encountered by students. The ALC's highest priority was the provision of an alternate accessible version of a tutorial, since it is difficult to make complex embedded web content accessible for everyone under every circumstance and an alternate version allows people to work with content in a way that suits their needs. 
For the evaluation, major database vendors were chosen through a scan of common vendors and platforms at universities, with input from collections colleagues. Some vendors were eliminated because they don't provide instructional tutorials on their websites. Twenty-five vendors were included in the study (see appendix 1). A large majority of the tutorials found were screencast or video tutorials; a few vendors provided recorded webinars, and a few provided interactive multimedia tutorials, mainly text captions or visuals with clickable areas or quizzes.

In total, 460 tutorials were evaluated for accessibility: 417 video, screencast, or interactive tutorials from twenty-foure vendors, and 41 recorded webinars from four vendors. If tutorials were available in more than one place, most commonly on both the vendor's website and YouTube, both locations were tested. If more than thirty tutorials were provided by a vendor, every other one was tested. If multiple formats of tutorial were available, such as screencasts and recorded webinars, each format was tested.

Testing from the perspective of people with visual impairments was a key focus. Other assistive technologies such as Kurzweil (for people who can see but have print-related disabilities) and Zoomtext (for enlargement) are widely used, but if webpages work well using screen-reading software intended for people with visual impairments, they also generally work using other kinds of assistive software. Tutorials were tested with two screen-reading programs used by people with visual impairments: NVDA (with Firefox), a free open source program, and JAWS (with Internet Explorer), a widely used commercial product. Both were used to determine whether any difficulties were due to the quirks of a particular software product or a result of inherent accessibility problems.

In addition, captions were evaluated to determine accessibility for people who are deaf or have hearing difficulties. People with visual or some physical impairments use the keyboard only, so all tutorials were tested without a mouse using solely the keyboard.

During testing, each task was tried three different ways within NVDA or JAWS before deciding that it couldn't be completed. If one of the three methods worked the task was marked as successfully completed. If a task could be completed successfully in one screen-reading program but not the other, it was marked as unsuccessful. Screen-reader support needs to be consistent across platforms, since people may be using a variety of types of assistive software.

\section{FINDINGS AND DISCUSSION}

Tutorials created by the same vendor nearly all used the same approach and had the same checklist results. This is positive, since consistency is important for accessibility and helps in navigation and ease of use.

None of the forty-one recorded webinars tested in this study were accessible. Webinars did not have player controls that were findable on the page by screen-reading software or usable by 
keyboard. None had captions, transcripts, or alternate accessible versions. Often webinars were quite long, with no clear structure and no cues to focus attention on the screen. Recorded webinars had almost no accessibility features and can't be recommended for use as accessible instructional materials in their current form.

None of the screencast or video tutorials tested were completely accessible, and all failed in at least one checklist item. Tutorials from some vendors, however, came close to meeting all checklist requirements. Overall, there were many positive accessibility features in the video and screencast tutorials. Most of these tutorials were findable and playable by screen reading software in some way, had video player controls usable by keyboard, had descriptive narration so people who can't see the screen can tell what is happening, had clear visuals and audio narration, used simple language, and were relatively short and focused in content.

The most accessible screencast or video tutorials were produced by the American Psychological Association (APA), American Theological Library Association (ATLA), Modern Language Association (MLA), and Ebsco. Their tutorials had many accessibility features and rated highly on the checklist. They included much less commonly found accessibility features, especially the use of visual and/or audio cues to focus the viewer's attention and the inclusion of accurate and properly synchronized closed captions. Visual cues are important for people with learning or attentionrelated disabilities, and help all viewers interpret and follow the video more easily. People who are deaf can't access the content without captions, and captions also help people who have English as a second language or are at public computers without headphones.

Tutorials from these vendors also had an alternate version or transcript available. As mentioned earlier, the highest-priority checklist item is the presence of an alternate accessible version, since it is difficult to design multimedia that works for people with all disabilities in all circumstances. People with disabilities may also have previous negative experiences with online multimedia and prefer to use an alternate format that they have had more success with.

In the case of these above-average vendors, the alternate accessible version was a transcript consisting of the video's closed captions, auto-generated by YouTube. Since the tutorials' narration was descriptive and the captions were accurate, the auto-generated transcripts are useful. However, the YouTube transcript is hard to find on the YouTube page. Also, most of these vendors had tutorials available both from their own websites and from YouTube, and none had alternate versions available on their own websites. Viewers requiring an alternate format would need to know to go to the YouTube site instead of the vendor site to find it.

Two other vendors also had quite accessible tutorials. IEEE's tutorials had the same positive accessibility features already mentioned. Tutorials were done in-house and presented through the vendor's site. While most tutorials presented on vendor sites were lacking in accessibility, IEEE's were well thought out from an accessibility perspective and usable by screen-reading software. These were the only tutorials tested where all interactivity, including pop-up screens, was easily 
usable and navigable by keyboard. The one accessibility issue was the lack of an alternate accessible version.

Elsevier's ScienceDirect tutorials took a different approach to accessibility than other vendors, or even than Elsevier's tutorials for other Elsevier products. The Science Direct tutorials were not accessible, but an alternate text version was available and people using screen-reader software were informed of this when they get to the tutorial page and were redirected to the text version. The ideal is to have one version that is accessible to everyone, but this approach is a good way to implement an alternate version if one accessible version isn't possible.

Screencasts or video tutorials from other vendors also have some good accessibility features, but these were balanced with serious accessibility problems. The main accessibility issues discovered include the following:

Alternate accessible versions: vendors who had captions and hosted their videos on YouTube did have auto-generated YouTube transcripts, but these were hard to find and were only useful if the captions were descriptive and accurate, which many were not. Apart from Elsevier's ScienceDirect tutorials, no vendors provided another format deliberately as an accessible alternative.

Captions: captions were missing or problematic in the tutorials of fourteen vendors, or 59 percent of the total. Five (21 percent) of vendors provided no captions at all for their tutorials. Nine (38 percent) had unedited, auto-generated YouTube captions, which are highly inaccurate and therefore don't provide usable access to the content for people who are deaf.

Tutorial not findable or playable on page: Twelve vendors (50 percent) had tutorials that were not findable on the webpage or playable for people using a keyboard or screenreading software. Most of these issues are with tutorials on vendor sites, which were often Flash-based or offered through non-YouTube third party sites like Vimeo. Four vendors (17 percent) offered access to their tutorials both through their own (inaccessible) website and YouTube, which is findable and playable by screen reading software. Eight (33 percent), however, only provided access through their (inaccessible) webpages, which means that people using a keyboard or screen reading software would not be able to use their tutorials.

No visual cues to focus attention: Eight vendors (33 percent) had no visual cues to focus attention in the video. Visual cues help people with certain disabilities focus on the essential part of the screen that is being discussed, help everyone more easily interpret and follow what is happening, and are known to help facilitate successful multimedia learning. 18 
Nondescriptive narration: Six vendors (25 percent) had tutorials with audio narration that didn't sufficiently describe what was happening on the screen. Narration needs to describe what is happening in enough detail so people who can't see the screen are not missing information available for sighted viewers.

Fuzzy visuals: Five vendors ( 21 percent) had tutorials with visuals that were fuzzy and hard to see. This makes viewing difficult for people with low vision, and challenging even for people with normal vision.

Fuzzy audio or background music: Three vendors (13 percent) had poor-quality audio narration or background music playing during narration. Background music is distracting for those with hearing difficulties and makes it more difficult to focus on what is being said. Eliminating extraneous sound also makes it easier for people to learn from multimedia. ${ }^{19}$

Tutorials consisting only of text captions: Three vendors (13 percent) had tutorials consisting of text captions with no narration. The text captions were not readable by screen-reading software, and no alternate accessible versions were provided. Providing narration in tutorials is recommended for accessibility, since it allows people who can't see the screen to access the content more easily, and has been shown to improve learning and recall over on-screen text and graphics alone..$^{20}$

\section{RECOMMENDATIONS AND CONCLUSIONS}

This study attempted to determine how accessible vendor-created database tutorials are, and whether academic librarians can use them instead of re-creating them locally. For recorded webinars, the answer is a clear no, since none were technically accessible for people using screenreading software.

For video or screencast tutorials, however, the answer less is clear. Results showed that many vendors created tutorials with positive features like clear visuals and audio, being short and focused on one main point, and using descriptive narration. However, technical accessibility was much less successful, with 59 percent of vendors omitting usable captions and 50 percent presenting tutorials that couldn't be found on the page or played by people using screen-reading software. These technical accessibility issues prevent people with hearing, vision, or some mobility impairments from using the tutorials at all. Although none of the tutorials studied met all the checklist criteria, some came close and could be used by librarians depending on local requirements, policies, and priorities for accessibility.

In part, this study found that the accessibility of many tutorials depends on how they are presented. Disappointingly, 50 percent of vendors had tutorials on their websites that were not findable or playable by people with disabilities. Many vendors, however, hosted tutorials on YouTube as well as their own site. In these cases, YouTube was always a more accessible option 
than the vendor site. YouTube itself is relatively accessible, with both pages and players that are navigable by keyboard and by screen-reading software. There are options for accessibility settings in YouTube, such as having captions display automatically, and more accessible third-party overlays are available for the YouTube player.

On vendor sites, there were more likely to be issues with Flash and an inability for people using screen-reading software or keyboards to find and play videos. Some vendors embed YouTube videos on their site. Even if the embedded videos are findable and playable, this method omits important accessibility features found on the YouTube page, such as the text transcript. The results of this study show that using YouTube where available is recommended. Further, linking to YouTube rather than embedding the video is preferred, unless a separate link to the transcript is made to provide an alternate accessible version.

Captions are another key accessibility problem identified in this study: nearly two-thirds had unusable captions. Often, auto-generated YouTube captions were present but were not usable. The presence of captions is not enough for accessibility; those captions need to be accurate and present the same content as the narration. YouTube auto-captioning does not generate captions that are accurate enough to be useful without manual editing. YouTube auto-generates transcripts from the captions, so if the captions are inaccurate the transcript will not be useful either. Editing YouTube auto-generated captions is necessary to ensure accessibility.

A few accessibility issues found in this study would be easy to improve with some thought during tutorial creation. Adding visual cues like arrows or highlighting to the screen to help people focus attention, or remembering that not everyone can see the screen while recording narration, can be easily achieved and would improve accessibility significantly.

Other issues would require more planning and effort to improve. Given the widespread technical accessibility problems identified in this study, it is particularly important for people creating tutorials to provide alternate formats that are accessible if tutorials themselves are not accessible. Almost no vendors do this currently, but it would have the most significant impact on accessibility for the broadest range of people. Adding usable captions is the second most important area for improvement. To provide access for people who are deaf, captions need to be added or autogenerated YouTube captions need to be edited for accuracy. Both alternate formats and captions require some thought and effort to implement but ensure that tutorials will meet accessibility requirements and be usable by everyone.

\section{NOTES AND BIBLIOGRAPHY}

1. Eamon Tewell, "Video Tutorials in Academic Art Libraries: A Content Analysis and Review," Art Documentation 29, no. 2 (2010): 53-61. 
2. Amanda S. Clossen, "Beyond the Letter of the Law: Accessibility, Universal Design, and Human-Centered Design in Video Tutorials," Pennsylvania Libraries: Research \& Practice 2, no. 1 (2014): 27-37, https://doi.org/10.5195/palrap.2014.43; Joanne Oud, "Improving Screencast Accessibility for People with Disabilities: Guidelines and Techniques," Internet Reference Services Quarterly 16, no. 3 (2011): 129-44, https://doi.org/10.1080/10875301.2011.602304; Kathleen Pickens and Jessica Long, “Click Here! (And Other Ways to Sabotage Accessibility)," Imagine, Innovate, Inspire: The Proceedings of the ACRL 2013 Conference (Chicago: ACRL, 2013), 107-12.

3. DeAnn Barnard-Brak, Lucy Lechtenberger, and William Y. Lan, "Accommodation Strategies of College Students with Disabilities," Qualitative Report 15, no. 2 (2010): 411-29.

4. Cyndi Rowland et al., "Universal Design for the Digital Environment: Transforming the Institution," Educause Review 45, no. 6 (2010): 14-28.

5. Peter Brophy and Jenny Craven, "Web Accessibility," Library Trends 55, no. 4 (2008): 950-72.

6. Kyunghye Yoon, Laura Hulscher, and Rachel Dols, "Accessibility and Diversity in Library and Information Science: Inclusive Information Architecture for Library Websites," Library Quarterly 86, no. 2 (2016): 213-29.

7. Ruth V. Small, William N. Myhill, and Lydia Herring-Harrington, "Developing Accessible Libraries and Inclusive Librarians in the 21st Century: Examples from Practice," Advances in Librarianship 40 (2015): 73-88, https://doi.org/10.1108/S0065-2830201540; John Carlo Jaeger, Paul T. Wentz, and Brian Bertot, "Libraries and the Future of Equal Access for People with Disabilities: Legal Frameworks, Human Rights, and Social Justice," Advances in Librarianship 40 (2015): 237-53; Yoon, Hulscher, and Dols, "Accessibility and Diversity in Library and Information Science: Inclusive Information Architecture for Library Websites."

8. Suzanne L. Byerley, Mary Beth Chambers, and Mariyam Thohira, "Accessibility of Web-Based Library Databases: The Vendors' Perspectives in 2007," Library Hi Tech 25, no. 4 (2007): 50927, https://doi.org/10.1108/07378830710840473; Kelly Dermody and Norda Majekodunmi, "Online Databases and the Research Experience for University Students with Print Disabilities," Library Hi Tech 29, no. 1 (2011): 149-60, https://doi.org/10.1108/07378831111116976; Jennifer Tatomir and Joan C. Durrance, "Overcoming the Information Gap: Measuring the Accessibility of Library Databases to Adaptive Technology Users," Library Hi Tech 28, no. 4 (2010): 577-94, https://doi.org/10.1108/07378831011096240.

9. Pickens and Long, "Click Here!”; Clossen, "Beyond the Letter of the Law”; Oud, "Improving Screencast Accessibility for People with Disabilities"; Nichole A. Martin and Ross Martin, "Would You Watch It? Creating Effective and Engaging Video Tutorials," Journal of Library \& 
Information Services in Distance Learning 9, no. 1-2 (2015): 40-56, https://doi.org/10.1080/1533290X.2014.946345.

10 . Byerley, Chambers, and Thohira, “Accessibility of Web-Based Library Databases.”

11. Tatomir and Durrance, "Overcoming the Information Gap."

12. Dermody and Majekodunmi, "Online Databases and the Research Experience for University Students with Print Disabilities."

13. Laura DeLancey, "Assessing the Accuracy of Vendor-Supplied Accessibility Documentation," Library Hi Tech 33, no. 1 (2015): 103-13, https://doi.org/10.1108/LHT-08-2014-0077.

14. Jodi B. Roberts, Laura A. Crittenden, and Jason C. Crittenden, "Students with Disabilities and Online Learning: A Cross-Institutional Study of Perceived Satisfaction with Accessibility Compliance and Services," Internet and Higher Education 14, no. 4 (2011): 242-50, https://doi.org/10.1016/j.iheduc.2011.05.004.

15. Kari L. Kumar and Ron Owston, "Evaluating E-Learning Accessibility by Automated and Student-Centered Methods," Educational Technology Research and Development 64, no. 2 (2015): 263-83, https://doi.org/10.1007/s11423-015-9413-6.

16. US Access Board, "Draft Information and Communication Technology (ICT) Standards and Guidelines," 36 CFR Parts 1193 and 1194, RIN 3014-AA37 (2015), https://www.accessboard.gov/attachments/article/1702/ict-proposed-rule.pdf.

17. Pickens and Long, "Click Here!"; Clossen, "Beyond the Letter of the Law”; Martin and Martin, "Would You Watch It?"; Oud, "Improving Screencast Accessibility for People with Disabilities."

18. See the Signaling Principle in Richard E. Mayer, Multimedia Learning, 2nd ed. (Cambridge: Cambridge University Press, 2009): 108-17.

19. See the Coherence Principle, Ibid., 89-107.

20. See the Modality Principle, Ibid., 200-220. 


\section{Appendix 1. List of Vendors}

1. ACM

2. Adam Matthew

3. Alexander St Press

4. APA

5. ATLA

6. ChemSpider

7. Cochrane Library (webinars only)

8. Ebsco

9. Elsevier

10. Factiva

11. Gale

12. IEEE

13. Lexis Nexis Academic (tutorials and webinars)

14. Marketline

15. MathSciNet

16. OVID/Wolters Kluwer (tutorials and webinars)

17. Oxford

18. Proquest (tutorials and webinars)

19. Pubmed

20. Sage

21. SciFinder

22. Standard \& Poor/NetAdvantage

23. Taylor and Francis

24. Web of Knowledge/Thompson Reuters

25. Zotero 


\section{Appendix 2. Tutorial Accessibility Evaluation Checklist}

\section{Functionality}

Equivalent alternate format(s) are provided

Transcript/test version

Audio

Other

Alternate formats provided are accessible

Alternate formats provided are findable on the page by screen reader

Screen reading software can find the video on the webpage

Screen-reading software can access and play the video

Video-player functions can by operated by keyboard/screen-reading software

Interactive content can be accessed and used by keyboard/screen-reading software

User has some control over timing (pause/rewind capability)

Alternate modes of presentation are available for all, meaning presented through text, visuals,

narration, color, or shape

Synchronized closed captions are available for all audio

Audio/narration is descriptive

\section{Usability}

User controls if/when the video starts (no auto play)

Video is easy to use by screen-reading software

Clear, high-contrast visuals and text

Clear, high-contrast audio (no background noise/music)

Uses visual cues to focus attention (e.g., highlighting, arrows)

Is short and concise

Is clearly and logically organized

Has consistent navigation, look, and feel

Uses simple language, avoids jargon, and defines unfamiliar terms

Explicit structure with sections, headings to give viewers context

Learning outcome/goal clearly outlined and content focused on outcome 